\title{
Mineral and Protein Contents of some Marine Algae from the Coastal Areas of Northern Sri Lanka
}

\author{
Rajeswary Mageswaran and S. Sivasubramaniam \\ Department of Chemistry, University of Jaffna, Jaffna, Sri Lanka
}

(Date of receipt: 24 April 1984)

(Date of acceptance: 10 October 1984)

\begin{abstract}
Protein and mineral contents of twenty-five species of marine algae from the coastal areas of Northern Sri Lanka are reported. Species such as Centroceros clavulatum, Ulva lactuca, Hypnea musciformis, Acanthophora delilei and Gracilaria edulis are found to have high protein content which are comparable to that in food materials such as cereals, eggs and fish. Algae such as Gracilaria edulis, Laurencia obtusa, Sargassum polycystum, Ulva lactuca and Gracilaria crassa and Thalasia hemprichi (angiosperm) have fairly large amounts of nitrogen, phosphorus and potassium and hence could be used as fertilizers.
\end{abstract}

\section{Introduction}

Marine algae and sea grasses are two of the important groups of marine plants occurring in the sea. They contain several inorganic elements and a complex mixture of organic substances synthesised from them. Due to the presence of these chemical substances marine algae find a variety of uses, some of which are briefly indicated below.

Marine algae are used ${ }^{3}$ to prepare alginic acid and agar. Fresh dried and processed seaweeds are utilised as human food 11, 14,15 in Japan, Indonesia, China, Philippines, India and other south east Asian countries. Seaweeds such as Porphyra, Ulva, Chlorella, Gracilaris and Chondrus are commonly used as ingredients for soups and as meat flavourings. ${ }^{11},{ }^{14},{ }^{15}$ Countries like Japan have large industries based on edible seaweeds. The algal carbohydrates are not easily digestible and hence the food value of the seaweeds depends on the minerals, trace elements, proteins and vitamins present in them. The seaweed meal is nutritious due to its high mineral and protein content. Seaweeds are used 11, 14, 15 to stock feed sheep and cattle in maritime districts.

Seaweeds contain reasonable quantities of nitrogen, phosphorus and potassium and they are extensively used, either directly or in the form of compost with cowdung as manure for vegetables in India. ${ }^{13}$ In seaweeds, the minerals and trace elements occur in water soluble form 13 and hence these could be easily taken up by the plants. The carbohydrates and other organic constituents of the seaweeds are reported 13 to increase the moisture holding capacity of soils. The marine algae are also a good source of potash and soda. 
During certain seasons fairly large quantities of seaweeds are found in the coastal areas of the Jaffna Peninsula. In order to make the best use of these seaweeds a knowledge of their chemical composition is essential. Therefore as an initial step, a study of the mineral, protein and vitamin contents of the seaweeds found in Northern Sri Lanka was started. Our results on the estimation of the moisture, ash and protein contents and of the amounts of some inorganic elements present in twenty five species of seaweeds are given below.

\section{Experimental Methods and Materials}

The seaweeds were collected from Mandaitivu, Nainativu and Keerimalai, washed well and air dried for three days. The moisture contents were determined by drying them in an oven at $105^{\circ} \mathrm{C}$ to constant weight. The ash contents were determined by ashing weighed quantities of samples at $450^{\circ} \mathrm{C}$ in a muffle furnace until constant weight was obtained. The total nitrogen was determined by the Kjeldhal method and protein content was calculated by multiplying the total nitrogen content by 6.25 .

\subsection{Preparation of Test Solutions}

Seaweed samples of known weights were ashed at $450^{\circ} \mathrm{C}$ and dissolved quantitatively in a known volume of $0.6 \mathrm{M} \mathrm{HCl}$ and the resulting solutions were used to determine the amounts of sodium, potassium, calcium, magnesium and trace elements.

\subsection{Determination of the amounts of Sodium and Potassium}

Sodium and potassium were determined using a Corning Model 400 flame photometer using appropriate interference filters. The flame intensities for sodium were corrected for interference by calcium by the standard addition method. ${ }^{17}$

\subsection{Determination of the amounts of Calcium and Magnesium}

The amount of calcium present in seaweeds was determined 12 by titrating a known volume of the test solution with standard EDTA with Patton-Reeder's indicator and using potassium cyanide as the masking agent.

The amount of magnesium present in seaweeds was estimated as follows: The total amount of magnesium and calcium present was determined by titrating known volumes of test solution with standard EDTA solution with Eriochrome Black $T$ as indicator and using potassium cyanide as masking agent. ${ }^{12}$ The amount of magnesium is obtained by subtracting the amount of calcium present from this value.

\section{- 2.4. Determination of Iron in Seaweeds}

The test solution was prepared in $0.6 \mathrm{M} \mathrm{HCl}$ as described above. The amount of iron present in the test solution was determined 8 by measuring the density of the colour produced by the addition of 1,10 - orthophenanthroline on a Corning model 252 colorimeter using a $490 \mathrm{~nm}$ filter. 


\subsection{Determination of Trace Elements}

Amounts of trace elements copper, manganous, nickel, zinc, cadmium, cobalt and chromium were determined using the test solution [prepared as described above] on a Varian Model 1275 atomic absorption spectrophotometer.

\subsection{Determination of the amount of Lead in Seaweeds}

Samples of known weight were subjected to wet oxidation with concentrated nitric acid and concentrated sulphuric acid by the reported procedure. 10 The resulting solution was used to prepare the test solution. The amount of lead present was determined 10 using Corning Model 252 colorimeter after complexing with dithizone.

\subsection{Determination of Phosphorss in Seaweeds}

Dried seaweed samples $\{1-2 \mathrm{~g} \mid$ were weighed accurately, ashed with magnesium nitrate, ${ }^{6}$ the ash was dissolved in $2\left(\mathrm{NH}_{4}\right)_{2} \mathrm{SO}_{4}(10 \mathrm{ml})$ and the solution made up to $50 \mathrm{ml}$. The amount of phosphorus present in this solution was determined ${ }^{6}$ by measuring the density of the colour produced on complexing with vanadomolybdate reagent on a Corning Model 252 colorimeter using 430, 470 and 490nm filters.

\subsection{Determination of Total Sulphur in Seaweeds}

The amount of total sulphur in seaweeds was determined by the reported method. ${ }^{6}$ Accurately weighed sample $(1-2 \mathrm{~g})$ of seaweed was treated with concentrated nitric acid $(10 \mathrm{ml})$ and the mixture was allowed to stand overnight at room temperature. The resulting mixture was evaporated to dryness and the residue was ashed in a muffle furnace at $450^{\circ} \mathrm{C}$. The ash was dissolved in a minimum volume of $25 \%$. nitric acid and the solution made up to $50 \mathrm{ml}$ in a volumetric flask. A known volume of this solution was treated with an excess of standard barium chloride. The unreacted barium chloride was determined by titration with standard EDTA. The amount of sulphate was estimated by the method of difference.

\subsection{Determination of the amount of Chloride in Seaweeds}

Accurately weighed powdered seaweed $(1.2 \mathrm{~g})$ was ashed at $450^{\circ} \mathrm{C}$ with an equal weight of sodium carbonate. The ash was dissolved in minimum amount of $25 \%$ nitric acid and the solution made up to $50 \mathrm{ml}$ in a volumetric flask. The amount of chloride present in this solution was determined by Vollard method.16

\section{Results and Discussion}

The amounts of moisture, ash, total nitrogen and protein found in twenty five species of the seaweeds from the coastal areas of Northern Sri Lanka are given in Table 1. The results show that some of the algae such as Gracilaria edulis, Gracilaria confervoides, Hypnea musciformis, Acanthophora delilei, Centroceros clavulatum and Ulva lactuca have between $13-26 \%$ protein and this amount is comparable or even 
somewhat higher than the reported ${ }^{4}$ protein content of food materials like cereals, eggs and fish. Algal proteins are reported ${ }^{11,14},{ }^{15}$ to have many of the essential aminoacids including iodine containing aminoacids and hence the above seaweeds, are a good source for protein rich food. Also, the above species of seaweeds could be added to foods which are deficient in protein. In India Gracilaria edulis is used ${ }^{11,14}, 15$ in the preparation of soup.

Table 1. Moisture, Ash, Nitrogen and Protein contents of Seaweeds. All values are expressed as $\mathrm{g} / 100 \mathrm{~g}$ of air dried samples of seaweeds

\begin{tabular}{|c|c|c|c|c|c|c|}
\hline Alga & Locality & $\begin{array}{l}\text { Date of } \\
\text { Collection }\end{array}$ & Moisture & Ash & Nitrogen & Protein \\
\hline \multicolumn{7}{|l|}{ a. Rhadophyta } \\
\hline 1. Gracilaria & Mandaitivu & 10.12 .82 & 3.69 & 38.46 & 2.28 & 14.25 \\
\hline edulis & Nainativu & 01.01 .83 & 13.9 & 24.86 & 1.89 & 11.81 \\
\hline $\begin{array}{l}\text { 2. Gracilaria } \\
\text { crassa }\end{array}$ & Mandaitivu & 10.12 .82 & 13.31 & 49.85 & 1.33 & 8.31 \\
\hline $\begin{array}{l}\text { 3. Gracilaria } \\
\text { confervoides }\end{array}$ & Keerimalai & 13.02 .83 & 10.07 & 8.80 & 2.30 & 14.38 \\
\hline 4. Laurencia obtusa & Mandaitivu & 10.12 .82 & 15.07 & 34.30 & 1.70 & 10.63 \\
\hline 5. Jania natalensis & Mandaitivu & 10.12 .82 & 3.21 & 69.79 & 0.75 & 4.69 \\
\hline 6. Hypnea & Mandaitivu & 10.12 .82 & 13.52 & 24.19 & 2.13 & 13.31 \\
\hline musciformis & Keerimalai & 13.02 .83 & 7.62 & 14.99 & 3.06 & 19.13 \\
\hline $\begin{array}{l}\text { 7. Acanthophora } \\
\text { delilei }\end{array}$ & Keerimalai & 13.02 .83 & 9.98 & 25.94 & 2.69 & 16.87 \\
\hline 8. Centraceros & Mandaitivu & 31.12 .82 & 16.66 & 29.29 & 1.99 & 12.44 \\
\hline clavulatum & Keerimalai & 13.02 .83 & 8.08 & 18.65 & 4.02 & 25.13 \\
\hline $\begin{array}{l}9 \text { Gelidiella } \\
\text { acerosa } \\
\text { b. Phaeophyta }\end{array}$ & Mandaitivu & 31.12 .82 & 14.12 & 12.07 & 1.67 & 10.44 \\
\hline 10. Padina pavonia & Mandaitivu & 10.12 .82 & 11.15 & 42.78 & 1.80 & 11.25 \\
\hline $\begin{array}{l}\text { 11. Turbinaria } \\
\text { omata }\end{array}$ & Mandaitivu & 31.12 .82 & 19.43 & 24.63 & 1.38 & 8.63 \\
\hline $\begin{array}{l}\text { 12. Pocockiella } \\
\text { variegata }\end{array}$ & Nainativu & 01.01 .83 & 13.37 & 19.82 & 1.18 & 7.38 \\
\hline $\begin{array}{l}\text { 13. Stoechospermum } \\
\text { marginatum }\end{array}$ & Mandaitivu & 13.02 .83 & 13.09 & 21.94 & 1.49 & 9.31 \\
\hline $\begin{array}{l}\text { 14. Cystophyllum } \\
\text { muricatum }\end{array}$ & Mandaitivu & 13.02 .83 & 13.84 & 35.14 & 1.45 & 9.06 \\
\hline $\begin{array}{l}\text { 15. Turbinaria } \\
\text { conoides }\end{array}$ & Nainativu & 01.01 .83 & 14.13 & 16.95 & 0.93 & 5.81 \\
\hline 16. Sargassum & Mandaitivu & 31.12 .82 & 17.07 & 35.51 & 1.61 & 10.06 \\
\hline polycystum & Nainativu & 01.01 .83 & 14.59 & 14.44 & 1.14 & 7.13 \\
\hline $\begin{array}{l}\text { 17. Sargassum } \\
\text { tenerrimum }\end{array}$ & Mandaitivu & 10.12 .82 & 16.03 & 31.17 & 1.56 & 9.75 \\
\hline $\begin{array}{l}\text { 18. Hormophysa } \\
\text { triquetra }\end{array}$ & Mandaitivu & 13.02 .83 & 13.06 & 46.91 & 0.91 & 5.69 \\
\hline
\end{tabular}


Table 1 (Contd.)

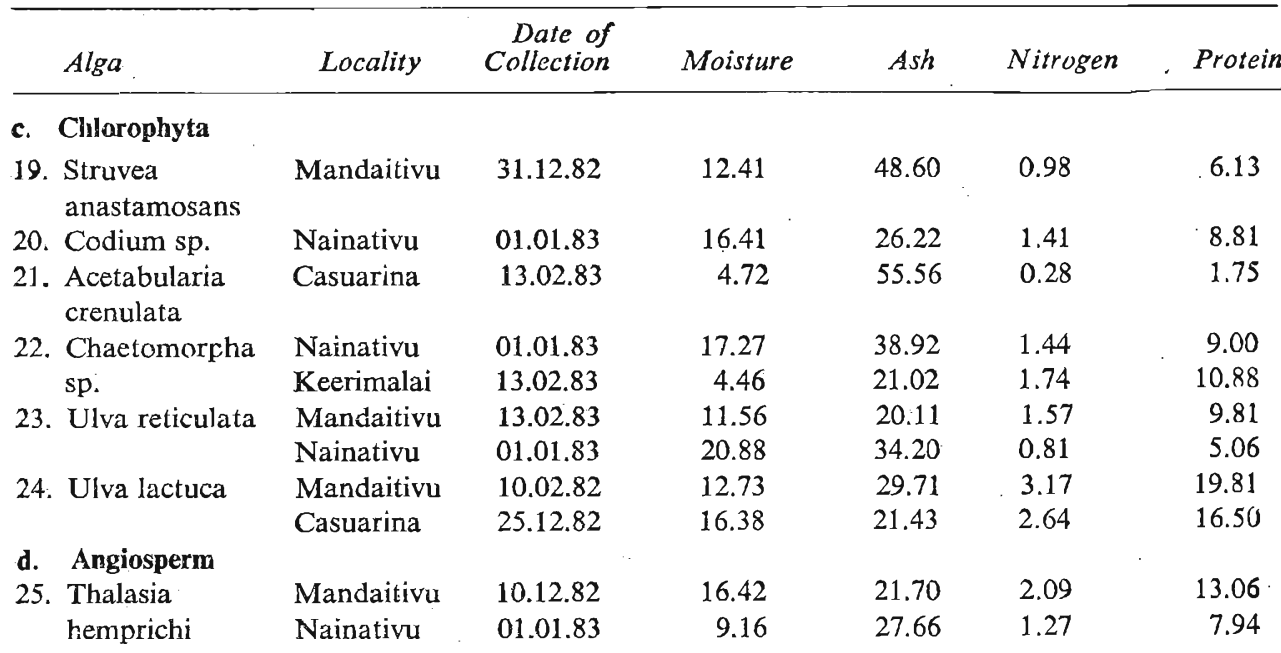

It is interesting to note that generally the seaweeds from the Keerimalai area have a higher protein content than those from the Mandaitivu coast, which in turn have a higher protein content than those from Nainativu coast.

Amounts of the minerals sodium, potassium, calcium and magnesium present in twenty five species of seaweeds were determined by the methods indicated above and our results are given in Table II. Gracilaria edulis, Gracilaria crassa, Laurencia obtusa, Turbinaria ornata and Sargassum tenerrimum have fairly large amount of potassium. Jania natalensis, Acetabularia crenulata, Padina pavonia, Sturvea anastamosans, Thalasia hemprichi, Pocockiella variegata, Laurencia obtusa, Hypnea musciformis and Ulva reticulata have reasonably large quantity of calcium. It is interesting to note that Jania natalensis, which is capable of accumulating calcium and depositing it in the form of a calcareous skeleton, has the highest amount of calcium. Jania natalensis, Chaetomorpha sp, Padina pavonia, Laurencia obtusa, Ulva reticulata and Thalasia hemprichi have more than $1 \%$ magnesium.

TARLe 2. Amounts of Sodium, Potassium, Calcium and Magnesium present in Seaweeds. All values are expressed in $\mathrm{g} / 100 \mathrm{~g}$ of air dried samples of seaweeds.

\begin{tabular}{|c|c|c|c|c|c|c|}
\hline Alga & Locality & $\begin{array}{l}\text { Date of } \\
\text { collection }\end{array}$ & Sodium & Potassium & Calcium & Magnesium \\
\hline \multicolumn{7}{|l|}{ a. Rhodophyta } \\
\hline $\begin{array}{l}\text { 1. Gracilaria } \\
\text { edulis }\end{array}$ & Mandaitivu & 10.12 .82 & 1.61 & 13.49 & 3.55 & 0.68 \\
\hline $\begin{array}{l}\text { 2. Gracilaria } \\
\text { crassa }\end{array}$ & Mandaitivu & 10.12 .82 & 0.75 & 7.63 & 2.02 & 0.23 \\
\hline $\begin{array}{l}\text { 3. Gracilaria } \\
\text { confervoides }\end{array}$ & Keerimalai & 13.02 .83 & 0.10 & 0.128 & 1.50 & $\begin{array}{l}0.69 \\
\text { (Contd.) }\end{array}$ \\
\hline
\end{tabular}


(Table 2 Contd.)

\begin{tabular}{|c|c|c|c|c|c|c|}
\hline Alga & Locality & $\begin{array}{c}\text { Date of } \\
\text { Collection }\end{array}$ & Sodium & Potassium & Calcium & Magnesium \\
\hline 4. Laurencia obtusa & Mandaitivu & 10.12 .82 & 1.22 & 4.32 & 4.99 & 1.35 \\
\hline 5. Jania natalensis & Mandaitivu & 10.12 .82 & 0.70 & 0.28 & 23.85 & 2.71 \\
\hline \multirow{3}{*}{$\begin{array}{l}\text { 6. Hypnea } \\
\text { musciformis } \\
\text { 7. Acanthophora } \\
\text { delilei }\end{array}$} & Mandaitivu & 10.12 .82 & 0.50 & 0.58 & 4.83 & 0.38 \\
\hline & Keerimalai & 13.02 .83 & 0.13 & 0.106 & 3.00 & 0.74 \\
\hline & Keerimalai & 13.02 .83 & 0.10 & 0.106 & 1.57 & 0.45 \\
\hline \multirow{2}{*}{$\begin{array}{l}\text { 8. Centroceros } \\
\text { clavulatum }\end{array}$} & Mandaitivu & 31.12 .82 & 1.20 & 3.26 & 2.53 & 0.46 \\
\hline & Keerimala1 & 13.02 .83 & 0.10 & 0.103 & 2.97 & 0.72 \\
\hline $\begin{array}{l}\text { 9. Gelidiella } \\
\text { acerosa }\end{array}$ & Mandaitivu & 31.12 .82 & 0.25 & 0.19 & 1.58 & 0.34 \\
\hline \multicolumn{4}{|l|}{ b. Phaeophyta } & & & \\
\hline 10. Padina pavonia & Mandaitivu & 10.12 .82 & 0.59 & 1.13 & 10.96 & 1.36 \\
\hline $\begin{array}{l}\text { 11. Turbinaria } \\
\text { ornata }\end{array}$ & Mandaitivu & 31.12 .82 & 1.81 & 6.30 & 2.12 & 0.28 \\
\hline $\begin{array}{l}\text { 12. Pocockiella } \\
\text { variegata }\end{array}$ & Nainativu & 01.01 .83 & 0.30 & 0.15 & 5.21 & 0.43 \\
\hline $\begin{array}{l}\text { 13. Stoechospermum } \\
\text { marginatum }\end{array}$ & Mandaitivu & 13.02 .83 & 0.25 & 1.10 & 2.08 & 0.07 \\
\hline $\begin{array}{l}\text { 14. Cystophyllum } \\
\text { muricatum }\end{array}$ & Mandaitivu & 13.02 .83 & 0.10 & 0.58 & 2.46 & 0.15 \\
\hline $\begin{array}{l}\text { 15. Turbinaria } \\
\text { conoides }\end{array}$ & Nainativu & 01.01 .83 & 0.49 & 1.68 & 2.28 & 0.35 \\
\hline \multirow{2}{*}{$\begin{array}{l}\text { 16. Sargassum } \\
\text { polycystum }\end{array}$} & Mandaitivu & 31.12 .82 & 2.62 & 3.93 & 3.15 & 0.56 \\
\hline & Nainativu & 01.01 .83 & 0.54 & 1.17 & 2.01 & 0.45 \\
\hline $\begin{array}{l}\text { 17. Sargassum } \\
\text { tenerrimum }\end{array}$ & Mandaitivu & 10.12 .82 & 0.82 & 5.97 & 3.06 & 0.84 \\
\hline $\begin{array}{l}18 \text { Hormophysa } \\
\text { triquetra }\end{array}$ & Mandaitivu & 13.02 .83 & 0.09 & 0.68 & 2.67 & 0.42 \\
\hline \multicolumn{7}{|l|}{ c. Chlorophyta } \\
\hline $\begin{array}{l}\text { 19. Sturvea } \\
\text { anastamosans }\end{array}$ & Mandaitivu & 31.12 .82 & 3.16 & 1.80 & 9.61 & 0.41 \\
\hline 20. Codium sp. & Nainativu & 01.01 .83 & 1.78 & 0.23 & 2.31 & 0.83 \\
\hline $\begin{array}{l}\text { 21. Acetabularia } \\
\text { crenulata }\end{array}$ & Casuarina & 13.02 .83 & 0.14 & 0.27 & 16.06 & 0.39 \\
\hline \multirow{2}{*}{$\begin{array}{l}\text { 22. Chaetomorpha } \\
\mathrm{sp} \text {. }\end{array}$} & Nainativu & 01.01 .83 & 1.71 & 0.81 & 2.40 & 1.94 \\
\hline & Keerimalai & 13.02 .83 & 0.21 & 0.10 & 2.88 & 1.27 \\
\hline \multirow[t]{2}{*}{ 23. Ulva reticulata } & Mandaitivu & 13.02 .83 & 0.31 & 0.31 & 4.82 & 0.48 \\
\hline & Nainativu & 01.01 .83 & 0.77 & 0.38 & 3.79 & 1.35 \\
\hline \multirow{2}{*}{ 24. Ulva lactuca } & Mandaitivu & 10.12 .82 & 0.85 & 1.85 & 2.44 & 0.17 \\
\hline & $\begin{array}{l}\text { Casuarina } \\
\text { Beach }\end{array}$ & 25.12 .82 & 0.96 & 2.25 & 3.60 & 0.25 \\
\hline \multirow{2}{*}{$\begin{array}{l}\text { 25. Thalasia } \\
\text { hemprichi }\end{array}$} & Mandaitivu & 10.12 .82 & 3.84 & 4.09 & 3.57 & 1.16 \\
\hline & Nainativu & 01.01 .83 & 0.57 & 0.41 & 7.46 & 0.84 \\
\hline
\end{tabular}


Our results on the colorimetric estimation of the amounts of iron and phosphorus are shown in Table III. It is seen that species such as Gracilaria edulis; Padina pavonia, Pocockiella variegata, Chaetomorpha sp. Sargassum polycystum and Jania natalensis are rich in iron. Also the seaweeds from the Nainativu and Mandaitivu areas generally have more iron content than those from Keerimalai area Gracilaria edulis, Gracilaria crassa, Gracilaria confervoides, Hypnea musciformis. Codium sp, Chaetomorpha sp. Ulva lactuca and Thalasia hemprichi have relatively large amounts of phosphorus.

TABle 3. Amounts of Iron and Phosphorus present in seaweeds. All values are expressed in $\mathrm{mg} / \mathrm{kg}$ of air dried samples of seaweeds.

\begin{tabular}{|c|c|c|c|c|}
\hline Alga & Locality & $\begin{array}{l}\text { Date of } \\
\text { Collection }\end{array}$ & Iron & Phosphorus \\
\hline \multicolumn{5}{|l|}{ a. Rhodophyta } \\
\hline \multirow{2}{*}{ 1. Gracilaria edulis } & Mandaitivu & 10.12 .82 & 405 & 764 \\
\hline & Nainativu & 01.01 .83 & 1240 & 989 \\
\hline 2. Gracilaria crassa & Mandaitivu & 10.12 .82 & 267 & 888 \\
\hline 3. Gracilaria confervoides & Keerimalai & 13.02 .83 & 262 & 1168 \\
\hline 4. Laurencia obtusa & Mandaitivu & 10.12 .82 & 674 & 514 \\
\hline 5. Jania natalensis & Mandaitivu & 10.12 .82 & 940 & 206 \\
\hline \multirow{2}{*}{ 6. Hypnea musciformis } & Mandaitivu & 10.12 .82 & 658 & 259 \\
\hline & Keerimalai & 13.02 .83 & 409 & 1338 \\
\hline 7. Acanthophora delilei & Keerimalai & 13.02 .83 & 102 & 225 \\
\hline \multirow{2}{*}{ 8. Centroceros clavulatum } & Mandaitivu & 31.12 .83 & 450 & 508 \\
\hline & Keerimalai & 13.02 .83 & 447 & 643 \\
\hline 9. Gelidiella acerosa & Mandaitivus & 31.12 .82 & 344 & 395 \\
\hline \multicolumn{5}{|l|}{ b. Pbaeophyta } \\
\hline 10. Padina pavonia & Mandaitivu & 10.12 .82 & 1266 & 477 \\
\hline 11. Turbinaria ornata & Mandaitivu & 31.12 .82 & 372 & 252 \\
\hline 12. Pocockiella variegata & Nainativu & 01.01 .83 & 1213 & 411 \\
\hline 13. Stoechospermum marginatum & Mandaitivu & 13.02 .83 & 134 & 608 \\
\hline 14. Cystophyllum muricatum & Mandaitivu & 13.02 .83 & 391 & 727 \\
\hline 15. Turbinaria conoides & Nainativu & 01.01 .83 & 306 & 472 \\
\hline \multirow[t]{2}{*}{ 16. Sargassum polycystum } & Mandaitivu & 31.12 .82 & 490 & 476 \\
\hline & Nainativu & 01.01 .83 & 373 & 469 \\
\hline 17. Sargassum tenerrimum & Mandaitivu & 10.12 .82 & 925 & 540 \\
\hline 18. Hormophysa triquetra & Mandaitivu & 13.02 .83 & 394 & 217 \\
\hline \multicolumn{5}{|l|}{ c. Chlorophyta } \\
\hline 19. Sturvea anastamosans & Mandaitivu & 31.12 .82 & 738 & 175 \\
\hline 20. Codium sp. & Nainativu & 01.01 .83 & 602 & 877 \\
\hline 21. Acetabularia crenulata & Casuarina & 13.02 .83 & 250 & 190 \\
\hline \multirow[t]{2}{*}{ 22. Chaetomorpha sp. } & Nainativu & 01.01 .83 & 1179 & 951 \\
\hline & Keerimalai & 13.02 .83 & 593 & 716 \\
\hline 23. Ulva reticulata & Mandaitivu & 13.02 .83 & 201 & 354 \\
\hline \multirow{2}{*}{ 24. Ulva lactuca } & Mandaitivu & 10.12 .82 & 219 & 1224 \\
\hline & Casuarina & 25.12 .82 & 542 & 633 \\
\hline \multirow[t]{2}{*}{ 25. Thalasia hemprichi } & Mandaitivu & 10.12 .82 & 392 & 1326 \\
\hline & Nainativu & 01.01 .83 & 655 & 1316 \\
\hline
\end{tabular}


The amounts of nitrogen, potassium and phosphorus present in selected species of seaweeds are shown in Table IV. It is interesting to note that Gracilaria edulis, Laurencia obtusa, Padina pavonia, Ulva lactuca, Thalasia hemprichi, Gracilaria crassa, Sargassum polycystum and Sargassum tenerimum have fairly large amounts of nitrogen, phosphorus and potassium and hence these could be used as fertilizers. Also other species which are rich in nitrogen, phosphorus or potassium could be mixed together to form a good fertilizer.

TABLE 4. The amounts of nitrogen, potassium and phosphorus present in selected species of seaweeds. All values are expressed in $\mathrm{mg} / \mathrm{kg}$ of air dried samples.

\begin{tabular}{|c|c|c|c|c|c|}
\hline Species & Locality & $\begin{array}{l}\text { Date of } \\
\text { Collection }\end{array}$ & Nitrogen & Phosphorus & Potassium \\
\hline 1. Gracilaria edulis & Mandaitivu & 10.12 .82 & 22800 & 764 & 134900 \\
\hline $\begin{array}{l}\text { 2. Gracilaria } \\
\text { confervoides }\end{array}$ & Keerimalai & 13.02 .83 & 23000 & 1168 & 1280 \\
\hline 3. Laurencia obtusa & Mandaitivu & 10.12 .82 & 17000 & 514 & 43200 \\
\hline \multirow[t]{2}{*}{ 4. Hypnea musciformis } & Mandaitivu & 10.12 .82 & 21300 & 259 & 5800 \\
\hline & Keerimalai & 13.02 .83 & 30600 & 1338 & 1060 \\
\hline $\begin{array}{l}\text { 5. Acanthophora } \\
\text { delilei }\end{array}$ & Keerimalai & 13.02 .83 & 26900 & 225 & 1060 \\
\hline \multirow{2}{*}{$\begin{array}{l}\text { 6. Centroceros } \\
\text { clavulatum }\end{array}$} & Keerimalai & 13.02 .83 & 40200 & 643 & 1030 \\
\hline & Mandaitivu & 31.12 .82 & 19900 & 508 & 32600 \\
\hline 7. Gelidiella acerosa & Mandaitivu & 31.12 .82 & 16700 & 395 & 1900 \\
\hline 8. Padina pavonia & Mandaitivu & 10.12 .82 & 18000 & 477 & 11300 \\
\hline $\begin{array}{l}\text { 9. Sargassum } \\
\text { polycystum }\end{array}$ & Mandaitivu & 31.12 .82 & 16100 & 476 & 39300 \\
\hline 10. Chaetomorpha sp. & Keerimaiai & 13.02 .83 & 17400 & 716 & 1000 \\
\hline \multirow[t]{2}{*}{ 11. Ulva lactuca } & Mandaitivu & 10.12 .82 & 31700 & 1224 & 18500 \\
\hline & Casuarina & 25.12 .82 & 24600 & 633 & 22500 \\
\hline 12. Thalasia hemprichi & Mandaitivu & 10.12 .82 & 20900 & 1326 & 40900 \\
\hline 13. Gracilaria crassa & Mandaitivu & 10.12 .82 & 13300 & 888 & 76300 \\
\hline 14. Turbinaria ornata & Mandaitivu & 31.12 .82 & 13800 & 252 & 63000 \\
\hline $\begin{array}{l}\text { 15. Sargassum } \\
\text { tenerrimum }\end{array}$ & Mandaitivu & 10.12 .82 & 15600 & 540 & 59700 \\
\hline
\end{tabular}

Seaweeds also contain trace elements. These arc a group of elements which are needed in infinitcly small amounts and these are very essential for the growth of plants and animals. Some of the important trace elements are $\mathrm{Fc}, \mathrm{Cu}, \mathrm{Zn}, \mathrm{Mn}$, Co, B and Mo. Some of the trace clements form complexes with enzymes and catalyse in metabolic reactions. The element copper is found in the enzyme polyphenol oxidase and the respiratory pigment of invertebrates, homocyanin. The enzymes such as tyrosinase, laccase and ascorbic acid oxidase use copper to catalyse reactions. ${ }^{1,9}$ Copper and cobalt are essential for health and productivity of animals. These two elements are associated with iron in the production of haemoglobin., ${ }^{1,9}$ 
The element cobalt, in addition to being an essential element of Vitamin $\mathrm{B}_{12}$ is important in the metabolism of sulphur containing aminoacids. Elements such as $\mathrm{Zn}, \mathrm{Mn}, \mathrm{Mo}, \mathrm{Cr}$ and $\mathrm{Cd}$ are also involved in the biological processes. ${ }^{13}$ Even though the trace elements have a vital function for human and animals they become toxic if present in relatively large amounts. The general recommended ${ }^{10}$ limits for some of the elements are given below.

$\begin{array}{llllll}\text { Element } & \mathrm{Pb} & \mathrm{Ni} & \mathrm{Cr} & \mathrm{Cu} & \mathrm{Zn} \\ \text { Recommended limit/ppm } & 2 & 100 & 100 & 20 & 50\end{array}$

The amounts of $\mathrm{Cu}, \mathrm{Zn}, \mathrm{Mn}, \mathrm{Ni}, \mathrm{Cd}$, Co and $\mathrm{Cr}$ present in twenty three species of marine algae were determined using Varian Model 1275 Atomic Absorption Spectrophotometer. The amount of lead present in these samples were determined colorimetrically after complexing with dithizone. No detectable amount of cobalt or chromium was found in any of these twenty three species. Our results, which are given in Table V, show that (i) Padina pavonia and Sturvea anastomosans have relatively large amounts of copper while the species Stoechospermum marginatum, Sargassum polycystum and Thalasia hemprichi have reasonable amounts of copper, (ii) the element zinc is found in relatively large amounts in Sturvea anastomosans, Dictyota species, Padina pavonia, Stoechospermum marginatum and Hypnea musciformis (iii) Manganese is found in relatively large amounts in red algae (iv) the species Sturvea anastomosans, Gracilaria edulis, Centroceros clavulatum, Jania natalensis, Hormophysa triquetra, Sargassum polycystum and Thalasia hemprichi have relatively high $\mathrm{Ni}$ content $(\mathrm{v})$ the element $\mathrm{Pb}$ is found in relatively large amount in Sturvea anastomosans, Gracilaria crassa, Gracilaria salicornia, Jania natalensis, Padina pavonia, Hormophysa triquetra and Sargassum polycystum and (vi) Cadmium is found in the range $1-4 \mathrm{ppm}$.

Cadmium and lead are non-nutritive toxic elements. Seaweed species analysed have low cadmium level (1-4 ppm). However the level of lead is rather high. The statutory limit for $\mathrm{Pb}$ in food is $2 \mathrm{ppm}$.

Sturvea anastomosans, Jania natalensis and Padina pavonia have relatively large amounts of the toxic elements and these may be avoided as nutrients and fertilizers. Also it is apparent from the Table $\mathbf{V}$ that the seaweeds from the Mandaitivu coast are richer in trace elements than those from Nainativu.

TABle 5. Amounts of Copper, Zinc, Manganous, Nickel, Lead and Cadmium present in seaweeds. All values are expressed as mg per $\mathrm{kg}$ (i.e. ppm) of air dried samples of seaweeds. (Date of collection is the same as in the Tables 1 \& 2).

\begin{tabular}{llllllll}
\hline Alga & Locality & $\mathrm{Cu}$ & $\mathrm{Zu}$ & $\mathrm{Mn}$ & $\mathrm{Ni}$ & $\mathrm{Pb}$ & $\mathrm{Cd}$ \\
\hline a. Rhodaphyta & & & & & & & \\
1. Gracilaria edulis & Mandaitivu & 5.3 & 9.2 & 397.3 & 24.0 & 8.3 & 3.3 \\
2. Gracilaria crassa & Nainativu & 6.0 & 6.4 & 76.3 & 12.9 & 7.0 & 3.9 \\
& Mandaitivu & 6.3 & 6.0 & 217.2 & 11.9 & 10.1 & 2.5 \\
(Contd.)
\end{tabular}


(Table 5 Contd.)

\begin{tabular}{|c|c|c|c|c|c|c|c|}
\hline$\therefore A \lg a$ & Locality & $\mathrm{Cu}$ & $Z n$ & $M n$ & $\mathrm{Ni}$ & $\mathrm{Pb}$ & $\overline{C d}$ \\
\hline 3. Gracilaria salicornia & $\begin{array}{l}\text { Mandaitivu } \\
\text { (13.2.83) }\end{array}$ & 3.4 & 6.6 & 62.0 & 15.5 & 9.9 & 1.3 \\
\hline 4. Laurencia obtusa & Mandaitivu & 5.2 & 6.4 & 129.7 & 14.3 & 8.6 & 2.2 \\
\hline 5. Hypnea musciformis & Mandaitivu & 2.7 & 11.9 & 149.6 & 14.5 & 8.7 & 1.3 \\
\hline 6. Centroceros clavalatum & Mandaitivu & 6.7 & 5.9 & 48.1 & 21.8 & 8.8 & 2.2 \\
\hline 7. Gelidiella acerosa & Mandaitivu & 2.7 & 7.3 & 102.8 & 12.3 & 5.7 & 2.4 \\
\hline 8. Jania natalensis & Mandaitivu & 3.0 & 6.9 & 87.6 & 32.2 & 19.3 & 3.1 \\
\hline 9. Padina pavonia & Mandaitivu & 40.8 & 12.4 & 211.9 & 21.5 & 18.4 & 3.9 \\
\hline $\begin{array}{l}\text { 10. Stoechospermum } \\
\text { marginatum }\end{array}$ & Mandaitivu & 11.6 & 10.1 & 21.4 & 13.6 & 9.3 & 1.3 \\
\hline 11. Cystophyllum muricatum & Mandaitivu & 8.7 & 8.3 & 31.6 & 17.1 & 8.3 & 2.1 \\
\hline 12. Dictyota sp. & Mandaitivu & 7.2 & 14.5 & 37.2 & 16.3 & 8.1 & 2.0 \\
\hline 13. Hormophysa tiquetra & Mandaitivu & 5.4 & 7.4 & 29.0 & 22.3 & 11.0 & 1.6 \\
\hline \multirow[t]{2}{*}{ 14. Sargassum polycystum } & Mandaitivu & 10.3 & 8.1 & 189.1 & 23.9 & 10.4 & 2.8 \\
\hline & Nainativu & 5.6 & 5.4 & 29.0 & 6.9 & 6.9 & 1.6 \\
\hline 16. Turbinaria ornata & $\begin{array}{l}\text { Mandaitivu } \\
\text { Mandaitivu }\end{array}$ & 4.9 & $\begin{array}{l}7.6 \\
4.5\end{array}$ & $\begin{array}{r}138.6 \\
279\end{array}$ & 13.6 & 9.3 & 2.4 \\
\hline 17. Pocockiella variegata & Nainativu & 5.0 & 6.9 & 63.5 & $\begin{array}{r}9.7 \\
16.2\end{array}$ & $\begin{array}{r}5.4 \\
101\end{array}$ & $\begin{array}{l}1.6 \\
25\end{array}$ \\
\hline 18. Turbinaria conoides & Nainativu & 3.1 & 3.0 & 22.6 & 7.6 & $\begin{array}{r}10.1 \\
7.4\end{array}$ & $\begin{array}{l}2.3 \\
2.1\end{array}$ \\
\hline \multicolumn{8}{|l|}{ c. Chlarophyta } \\
\hline 19. Sturvea anastamosans & Mandaitivu & 20.7 & 20.2 & 110.2 & 37.3 & 18.5 & 2.4 \\
\hline 20. Ulva reticulata & Mandaitivu & 3.6 & 5.9 & 74.0 & 10.4 & 3.9 & 2.0 \\
\hline 21. Chaetomorpha sp. & Nainativu & 6.9 & 8.4 & 36.1 & 22.8 & 10.1 & 1.7 \\
\hline $\begin{array}{l}\text { 22. Codium sp. } \\
\text { d. Angiosperm }\end{array}$ & Nainativu & 4.5 & 10.4 & 45.1 & 9.7 & 9.3 & 4.3 \\
\hline 23. Thalasia hemprichi & Mandaitivu & 10.8 & 7.4 & 51.3 & 25.1 & 8.5 & 2.5 \\
\hline & $\lambda$ & 0.4 & 7.3 & 70.9 & 19.8 & 13.4 & 2.5 \\
\hline
\end{tabular}

Chloride ion which is a micronutrient and sulphur which is a macronutrient $^{2}$ are also found in seaweeds. Table VI gives the values obtained for the amounts of ionic chloride and total sulphur present in some seaweeds.

TABLE 6. Amounts of ionic chloride and total sulphur present in seaweeds. All values are expressed in $\mathrm{g} / 100 \mathrm{~g}$ of air dried seaweeds.

\begin{tabular}{lllll}
\hline Alga & Locality & $\begin{array}{l}\text { Date of } \\
\text { Collection }\end{array}$ & $\begin{array}{l}\text { Ionic } \\
\text { Chloride }\end{array}$ & $\begin{array}{l}\text { Total } \\
\text { Sulphur }\end{array}$ \\
\cline { 4 - 5 } a. Rhodophyta & & & \\
1. Centroceros clavulatum & Mandaitivu & 31.12 .82 & 6.43 & 2.02 \\
2. Gracilaria edulis & Mandaitivu & 10.12 .82 & 3.88 & 4.13 \\
b. Phaeophyta & Nainativu & 01.01 .83 & 1.58 & 2.79 \\
3. Sargassum polycystum & Mandaitivu & 31.12 .82 & 7.95 & 1.03 \\
4. Sargassum tenerrimum & Mandaitivu & 10.12 .82 & 7.23 & 1.71 \\
5. Pocockiella variegata & Nainativu & 01.01 .83 & 0.61 & .50 \\
6. Stoechospermum marginatum & Mandaitivu & 13.02 .83 & 7.16 & 1.06 \\
c. Chlorophyta & Nainativu & 01.01 .83 & 5.88 & 3.65 \\
7. Codium sp. & Nainativu & 01.01 .83 & 8.07 & 4.03 \\
8. Chaetomorpha sp. & & & & \\
d. Angiosperm & Mandaitivu & 10.12 .82 & 5.34 & 0.58 \\
9. Thalasia hemprichi & Nainativu & 01.01 .83 & 2.67 & 0.49 \\
\hline
\end{tabular}

Our results show that Pocockiella variegata and Gracilaria edulis have relatively low amounts of ionic chloride. The species Thalasia hemprichi, Pocockiella variegata, Sargassum polycystum and Stoechospermum marginatum have relatively low amounts of sulphur. 


\section{Conclusion}

Some species of seaweeds such as Centroceros clavulatum, Gracilaria edulis, Gracilaria confervoides, Acanthophora delilei and Ulva lactuca have relatively large amounts of protein and could be used for fortifying food items deficient in protein. Seaweeds such as Gracilaria edulis, Laurencia obtusa, Padina pavonia, Ulva lactuca, Thalasia hemprichi, Gracilaria crassa, Sargassum polycystum and Sargassum tenerrimum have fairly large amounts of the elements nitrogen, phosphorus and potassium and these could be used as fertilizers. Gracilaria edulis and Gracilaria crassa also have relatively large amounts of the trace elements. Attempts should be made to cultivate the above species of algae so that they could be profitably used.

\section{Acknowledgements}

The authors wish to thank Prof. S. Balasubramaniam (Department of Botany, University of Peradeniya) and Dr. A. Sivapalan (Department of Botany, University of Jaffna) for helping in the collection and identification of seaweeds. They also wish to thank Professor R. S. Ramakrishna (Head, Department of Chemistry, University of Colombo) for facilities provided for the measurement of atomic absorption spectra. This work was supported by financial assistance from the Natural Resources, Energy and Science Authority of Sri Lanka.

\section{References}

1. Anderson, A. J. \& Underwood, E. J. (1959) Sci. Am. 97.

2. Bidwell, R. G. S. Plant Physiology. Collier Macmillan Publishers London p 262.

3. Dantanarayana, A. P., Savitri Kumar, N., Sultan Bawa, M. U. S. \& Balasubramanlam, S. (1981). J. Natn. Sci. Coun. Sri Lanka 9, and references cited therein.

4. Gopalan, Ramasastri \& Balasubramaniam, (1971). Nutritive values of Indian Foods The National Nutrition Institute, Hyderabad. (ICMR).

5. Holak, Walter \& Co. (1980). J. Assoc. Anal. Chem. 63, 485.

6. Jackson, M. L. (1973). Soil Chemical Analysis, Prentice-Hall of India Ltd., New Delbi, pp 151-154, 336, 389 .

7. Mageswaran, R. \& Sivasubramaniam, S. (1983). Proc. Cey. Assoc. Adv. Science. Part, I 39, 68.

8. Mageswaran, R., Sivasubramaniam, S. \& Somasundaram, K. (1983). Proc. of twelfth Ann Sess. I. Chem. C. Part I, 14.

9. McElroy, W. D. \& Swanson, C. P.(1953) Sci. Am. 22.

10. Pearson, D. (1976). The Chemical Analysis of food., Churchill Livingstone, Edinburgh, London and New York, pp 9, 84-86, 381.

11. Proc. Semi, Sea Salt and Plants (1967 \& 1970). CSMORI. Bhavangar.

12. Safar, D. N., (1974). Technology 11, pp 420-422 and Chenery, E. M. The Analyst (1964) 89 pp 365-367.

13. Seaweed Manure for perfect soil and smiling fields. Salt Res. Indust. (1964) 6, 1.

14. Second International Seaweed symposium (1956) edited by Trygue. Braarud and Sorensen. N. A., Pergamon Press, London and New York.

15. TRessler, D. K. Marine products of commerce, Chemical Catalog Co., New York.

16. Vogel, A. I. Quantitative Inorganic analysis, Third Edition (1961), Lowe and Brydone Ltd. p 266.

17. Willard, Hobart, H., Meririt, Lynne, L. \& Dean, John, A. Instrumental Methods of Analysis, Van Nostrand Reinhold Company, 342. 